\title{
NOUVELLES TENDANCES DANS LA DIDACTIQUE DE LA CHIMIE ET RÔLE DU COMITÉ POUR L'ENSEIGNEMENT DE LA CHIMIE DE L'IUPAC
}

\author{
CHASTRETTE, $M$. \\ LIRDIS - Université Lyon I, 43 Bd du 11 Novembre 1918 - 69622 Villeurbanne Ceđex * France.
}

\section{SUMMARY}

We call attention to the enormous spreading undergone by the didactic of chemistry in the last few years and comment on some of the trends that are significant in this field from the point of view of the author. We also comment on some of the projects developed by the committee for teaching chemistry in the IUPAC.

La didactique de la Chimie a connu récemment des développements importants et se trouve dans une phase d'expansion rapide. Il est bien présomptueux, dans ces conditions, de tenter de discerner les grandes tendances de ce mouvement et le point de vue que j'exposerai est à la fois partiel et partial. Il reflète les domaines d'intérêt du CTC de l'IUPAC et les miens propres. De plus I'évolution de la Didactique de la Chimie doit être replacée dans le cadre plus général des didactiques des Sciences.

Il est pourtant possible, à mon avis, de distinguer deux tendances vers une plus grande diversité.

La première tendance est l'internationalisation des recherches en didactique et des innovations significatives.

Au niveau de la recherche en Didactique, la Chimie participe au mouvement général des Didactiques des Sciences. Ainsi de nouveaux journaux périodiques sont apparus, en complément de ceux d'Amérique du Nord, en Australie et en Europe avec par exemple l'European Journal of Science Education, devenu récemment l'International Journal of Science Education.

De nouveaux groupes de recherche sont apparus dans de nombreux pays, en Europe, en Israël, en Asie du SudEst, en Amérique Latine. Un sondage sur les articles parus en 1988 dans l'International Journal of Science Education montre que 54 articles ont été écrits par des auteurs appartenant à 19 pays différents. On trouve ainsi 13 articles provenant du Royaume Uni, 7 d'Australie, 6 des USA et d'Israël, 4 de RFA et de France. Sur les 54 articles, 10 relèvent de la Chimie et proviennent de plusieurs pays. On constate aussi l'existence de collaborations de plus en plus fréquentes entre des équipes de plusieurs pays.

La même tendance est évidente au niveau des innovations et des recherches-actions. Alors que les pays anglophones et particulíèrement le Royaume Uni continuent à être très productifs, iI apparait un peu partout des groupes dynamiques qui conquièrent peu à peu leur autonomie. Un facteur très importantest le développement de réseaux qui couvrent toutes les régions du monde, pour ceux qui dépendent du Comité pour l'Enseignement de la Chimie de l'IUPAC, et principalement les pays développés pour le réseau sur l'enseignement de la Chimie assisté par ordinateur.

La deuxieme tendance est la diversification des domaines étudiés et des méthodes utilisées.

Il y a une vingtaine d'années les réflexions et les innovations étaient surtout centrées sur le contenu de l'enseignement. En effet, la Chimie avait été longtemps enseignée en 
insistant sur les aspects descriptifs et une réaction vigoureuse a poussé à mettre l'accent sur les aspects plus théoriques pour intéresser les étudiants et leur donner une formation plus large. On a vu ainsi paraître une série de manuels de Chimie à grand succès qui avaient comme point commun de comporter le mot «Principies» dans leur titre (figure 1).

lïigure 1

Caractéristiques de quelques livres publiés entre 1966 et 1968.

\begin{tabular}{|c|c|c|}
\hline Année d'édition & Auteurs & Titres \\
\hline 1966 & Sienko et Plane & $\begin{array}{l}\text { Chemistry : Principles } \\
\text { and Properties }\end{array}$ \\
\hline 1966 & Masterton et Slovinski & Chemical Principtes \\
\hline 1967 & Gray et Haight & $\begin{array}{l}\text { Basic Principles of } \\
\text { Chenistry }\end{array}$ \\
\hline 1968 & Dickerson, Gray, Haight & Chemical Principles \\
\hline
\end{tabular}

Dans les toutes dernières années une contre-réaction s'est amorcée dans plusieurs pays (dont le Canada et I'Australie), tendant à augmenter l'importance de la Chimie dite descriptive dans les curriculum. Certains prétendent qu'à l'heure actuelle on demande aux étudiants de mémoriser aussi bien les faits que les théories.

Le problème des contenus est rendu encore plus difficile par le souci légitime de faire passer dans l'enseignement les toutes dernières déccuvertes de la chimie de l'état solide ou des macromolécules par exemple. La Chimie telle qu'elle est enseignée à l'Université apparaît assez vieillotte, contrairement à la Biologie.

D'une manière très générale les grands projets đe recherche tels que le projet Global Change de I'ICSU fournissent des résultats scientifiques de qualité, d'un intérêt considérable pourl'humanité mais aussi assez peu exploités (et difficilement exploitables) pour l'enseignement. J'exposerai brièvement, plus loin, les efforts faits dans le cadre de l'ICSU et de l'IUPAC pour utiliser la masse énorme de ces résultats qui sont obtenus en mettant en jeu des budgets importants.

Alors que les contenus sont l'objet de vives controverses, les didacticiens de la chimie s'intéressent de plus en plus aux apprenants eux-mêmes. Cet intérêt se traduit par un flux important de publications sur les représentations des élèves (misconceptions, préconceptions, etc.), sur la résolution de problèmes, sur le traitement de l' information, sur la signification des travaux pratiques, pour ne citer que quelques uns des domaines étudiés.

Lors du dernier Congrès International sur l'Education en

Chimie (10th International Conference on Chemical Education) qui s'est tenu à Waterloo (Canada) à la fin du mois d'août, les communications par affiches ont dû être regroupées dans plusieurs rubriques telles que : compétitions enchimie (ex: Olympiades), équipement produit localement, attitudes envers l'industrie, High School Chemistry, formation des professeurs, stratégies pédagogiques, etc. Toutes ces communications ne présentent pas des recherches mais leur qualité me semble s'améliorer d'un congrès à l'autre.

Je ne pourrai malheureusement présenter dans le cadre de cet exposé que quelques exemples, qui m'ont semblé significatifs, de ces activités foisonnantes.

\section{LES RECHERCHES SUR LES REPRÉ- SENTATIONS: L'EXEMPLE DU CONCEPT D'EQUILIBRE EN CHIMIE}

Cet exemple a été choisi parmi de nombreux autres parce qu'il illustre bien les deux tendances citées plus haut. Les données sont tirées principalement d'un travail de Pereira (1986) qui analyse les principales publications sur le concept d'équilibre depuis 1966.

Les représentations observées semblent dépenđre peu des systèmes d'enseignement et du niveau des étudiants. Elles varient peư avec l'âge, ce qui indique qu'elles sont plutôt tenaces. La figure 2 indique les principaux domaines pour lesquels des représentations ont été repérées. Il est clair que le concept d'équilibre en chimie a été abordé sous des angles bien différents. Dans plusieurs cas des suggestions pratiques pour I'enseignementont été formulées à partir de ces recherches.

\section{Figure 2}

Domaines d'étude des conceptions erronées de l'équilibre chintque d'après Pereira (1986)

Signification des concentrations

Visualisation de l'équilibre

Signification du signe $\leftrightharpoons$

Distinction entre vitesse et avancement (de la réaction)

Signification du rendernent

Effet des catalyseurs

Energie d'activation ef équilíbre

Signification de la constante d'équilibre

Signification des lois de déplacement

Calcul des concentrations

Principe de Le Chatelier

Equilibres en phase liquide/gazeuse

Equilibres simultanés

La figure 3 illustre l'internationalisation des recherches et la diversification des méthodes d'études. En effet, les cinq publications partes entre 1966 et 1978 sont liées aux systèmes anglais et nord-américain mais parmi les cinq publications parues entre 1978 et 1986 trois sont relatives à des systèmes différents, en vigueur dans le Sud de l'Europe. 
Sides tests divers continuent à être employés, les publications récentes font appel aux entretiens individuels, aux commentaires libres, à l'autoévaluation, en combinant souvent plusieurs méthodes.

Il est prévisible que dans l'avenir on cherchera à prendre en compte d'une part les aspects expérimentaux de l'équilibre chimique et d'autre part ses relations avec le concept d'équilibre dans d'autres disciplines.

Figure 3

Analyse succincte de quelques publications sur les représentations de l'équilibre chimique d'après Pereira (1986).

\begin{tabular}{|c|c|c|c|c|c|}
\hline Auteurs & Date & $\begin{array}{l}\text { Nivears } \\
\text { Pays }\end{array}$ & Nbre & $\begin{array}{l}\text { Problèmes } \\
\text { étudiés }\end{array}$ & $\begin{array}{l}\text { Méthodes } \\
\text { utilisées }\end{array}$ \\
\hline Driscoll & 1996 & $\begin{array}{l}\text { Universile } \\
\text { Australie }\end{array}$ & 450 & Equil. chim. & $\mathrm{QCM}$ \\
\hline Doran & 1972 & $\begin{array}{l}\text { École élém. } \\
\text { USA }\end{array}$ & 253 & $\begin{array}{l}\text { équilibre el } \\
\text { théor. corpusc. }\end{array}$ & tests \\
\hline Mc Donald & 1973 & $\begin{array}{l}\text { higher grade } \\
\text { Ecosse }\end{array}$ & 255 & $\begin{array}{l}\text { équil. chim. } \\
\text { et origine des } \\
\text { représentations }\end{array}$ & $\begin{array}{l}\mathrm{QCM}+ \\
\text { justification }\end{array}$ \\
\hline Mc Donald & 1973 & $\begin{array}{l}\text { 6th yr stud. } \\
\text { Ecosse }\end{array}$ & 98 & $\begin{array}{l}\text { Equilibre et } \\
\text { thermodyn. }\end{array}$ & tests \\
\hline Wheeler & 1978 & $\begin{array}{l}\text { 12ème an. } \\
\text { Canada }\end{array}$ & 99 & $\begin{array}{l}\text { équilibre et } \\
\text { théor. Piaget }\end{array}$ & $\begin{array}{l}\mathrm{QCM}+ \\
\text { test Piaget }\end{array}$ \\
\hline Lorenzo & $198 !$ & $\begin{array}{l}2 \text { aire } \\
\text { Espagne }\end{array}$ & 138 & $\begin{array}{l}\text { classification } \\
\text { et fréquence } \\
\text { des répresent. }\end{array}$ & $\begin{array}{l}\text { lests } \\
+ \text { labo }\end{array}$ \\
\hline Vincent & 1981 & $\begin{array}{l}\text { Université } \\
\text { Angletere }\end{array}$ & 73 & $\begin{array}{l}\text { Equitibre et } \\
\text { volumétrie }\end{array}$ & $\begin{array}{l}\text { questions } \\
\text { commentaires }\end{array}$ \\
\hline Pereira & 1981 & $\begin{array}{l}2 \text { aire } \\
\text { Portugat }\end{array}$ & 300 & $\begin{array}{l}\text { difficultés } \\
\text { d'apprentiss. }\end{array}$ & $\begin{array}{l}\text { test avec } \\
\text { autoévaluation } \\
+ \text { questionnaire } \\
\text { professeurs }\end{array}$ \\
\hline Hackling & 1985 & $\begin{array}{l}2 \text { aire } \\
\text { Australie }\end{array}$ & 30 & $\begin{array}{l}\text { équilibre et } \\
\text { Ptincipe de } \\
\text { Le Chatelier }\end{array}$ & entretiens \\
\hline Cros & 1986 & $\begin{array}{l}\text { Uriversité } \\
\text { France }\end{array}$ & 140 & $\begin{array}{l}\text { difficultés } \\
\text { d'apprentiss. }\end{array}$ & $\begin{array}{l}\text { entretiens + } \\
\text { questionnaire }\end{array}$ \\
\hline
\end{tabular}

\section{RÉSOLUTION DE PROBLÈMES EN CHIMIE}

Beaucoup de travaux ont été consacrés, en chimie comme ailleurs, à la résolution de problèmes. L'approche đes chimistes est souvent très utilitaire et montre une grande diversité.
Les approches algoritmiques occupent une part encore importante mais heureusement en diminution. Frazer et son groupe ont analysé les démarches des étudiants et proposé des méthodes d'apprentissage applicables aussi dans l'industrie chimique. Tout récemment Pestana (1989) a analysé très en détail les causes d'erreurs et leurs implications pour l'enseignement.

Kempa a étudié les relations entre les capacités de résolution de problèmes et les réseaux conceptuels et, plus récemment, analysé les erreurs commises par les Élèves.

Le groupe de I'Université de Valence (Gil et Martínez Torregrosa (1983) et en particulier Furió et Hernández (1987) pour la Chimie) part d'une critique de l'efficacité des approches classiques. Ils proposent de remplacer les énoncés habituels par des situations ouvertes visant à développer la créativité et réduire les taux d'échecs.

Bien que ces approches aient fourni des résultats très intéressants et méritent d'être présentées en détail je ne les discuterai pas ici faute de temps et je me limiterai aux aspects relatifs au traitement de l'information.

\section{Capacités et demandes dans la résolution đe problèmes}

Plusieurs travaux importants abordent les aspects quantitatifs de la manipulation de l'information lors de la résolution d'un problème de chimie.

La difficulté d'un problème augmente, comme attendu, avec le nombre d'étapes nécessaires pour le résoudre. Frazer et Sleet (1984) et Lazonby et a1. (1982) ont montré que parmi lesétudiants qui disposent de l'information et des connaissances nécessaires pour traiter correctement les étapes d'un problème prises une par une, la moitié seulement est capable de traiter le problème dans son ensemble.

Un résultat semblable a été obtenu pour des problèmes d'appariement (entre des individus, des voitures, des couleurs, comme par exemple : Paul possède une voiture bleue qui n'est pas une Renault, Pierre a une Volkswagen, etc.) où l'on peut faire varier le nombre d'appariements à considérer. Polich et Schwartz (1974) ont montré que le pourcentage d'échecs dans ce genre de problèmes passe de $20 \%$ pour 10 appariements à $30 \%$ pour 15 à 20 appariements, $70 \%$ pour 25 et $90 \%$ pour 35 appariements.

Des travaux plus récents s'appuient sur l'iđée qu'il existe une limite à la capacité de notre mémoire de travail qui peut être surchargée si elle doit traiter trop d'information à la fois. La capacité limite semble être de $7 \pm 2$ unités d'information, en notant que l'unité d'information n'est pas nécessairement la même pour tous les sujets. Si cette capacité limite joue un rôle on doit s'attendre à ce que les problèmes soient d'autant plus diffíciles à résoudre qu'ils mettent en jeu plus d'information. Niaz (1987, 1989) utilise les règles de Case et de Pascual-Leone pour définir la M-demande d'un problème, c'est à dire le nombre d'unités d'information à manipuler pour le résoudre, 
Johnstone et El Banna (1986) montrent que les prédictions sont vérifiées pour un échantillon de 20000 candidats aux examens du Scottish Exam Board. L'indice de facilité des problèmes chute assez brutalement pour une $\mathrm{M}$ demande comprise entre 5 et 6 (figure 4 ).

Figure 4

Relations entre l'indice de facilité et la M-demande.

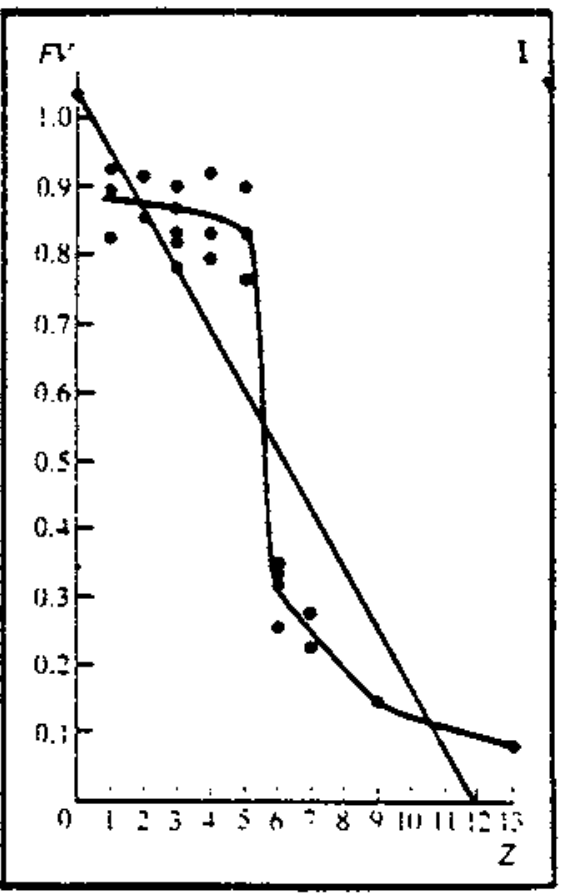

Il est remarquable que la courbe ne tende pas vers zéro, comme on devrait l'attenđre mais plutôt vers 0,1 . Cependant une population nombreuse et variée a certainement toute une gamme de capacités limites de traitement de l'information, de sorte que la courbe obtenue ne donne qu'une vue générale.

Il est intéressant de tester 1'hypothèse selon laquelle les étudiants de capacité connue ne peuvent pas résoudre des problèmes de $\mathrm{M}$-demande trop élevée. Pour pouvoir le faire il faut mesurer la M-capacité des étudiants. It existe pour cela plusieurs tests tels que le DBT (digit backwards test) ou le FIT (figure intersection test). Le DBT consiste à demander au sujet d'écrire ou de dire à I'envers une série de chiffres qu'on lui a lue. Le FIT consiste à reconnaître les parties communes à plusieurs figures partiellement superposées. Les deux mesures sont en assez bon accord (Johnstone et El Banna 1986).

Niaz (1989) utilise 23 questions de chimie dont la Mdemande varie de quatre à sept, sur un échantillon de 55 étudiants de première année d'Université au Vénézuela. Il trouve que les performances des étudiants de faible Mcapacité diminuent comme attendu lorsque la M-demande des problèmes augmente. Ainsi les étudiants de $\mathbf{M}$ capacité égale à 5 ont un score moyen de $44 \%$ pour les problèmes de $\mathrm{M}$-demande égale à 4 mais de $19 \%$ seulement si la M-demande est égale à 7.

Il apparaît aussi que beaucoup d'étudiants n'utilisent pas à plein leur M-capacité.

Niaz (1988) utilise une capacité fonctionnelle Mf qu'ì mesure aussi par le test FIT mais en limitant la durée du test. Une étude sur 100 étudiants de première année de ecole 1

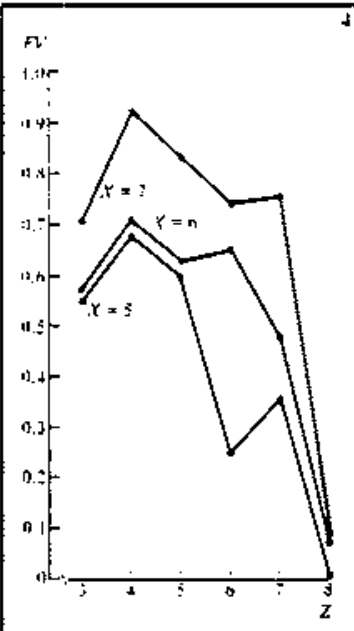

école 2

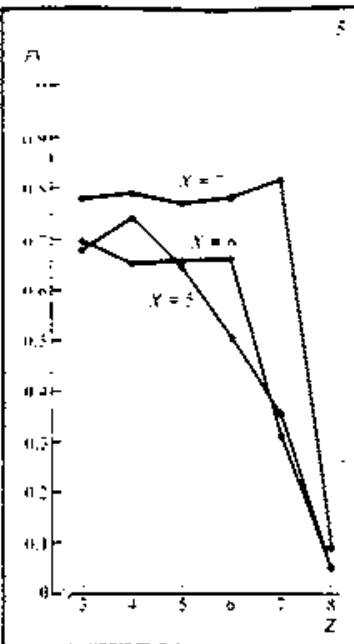

Université

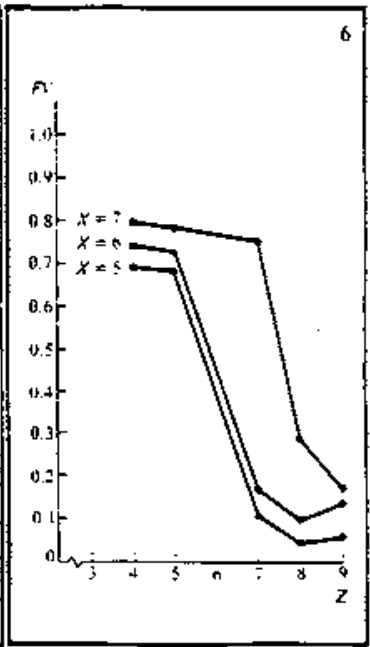


l'Université confirme que les performances dépendent comme attendu de la Mf et de la M-demande. Il apparaît que les corrélations entre $\mathrm{Mf}$ et les performances s'améliorent lorsque la M-demande augmente.

Johnstone et El Banna (1986) montrent que les élèves de $\mathrm{M}$-capacité égale à $\mathrm{Z}$ résolvent bien les problèmes de $\mathrm{M}$ demande inférieure à $Z$ mais mal ceux qui ont une $M$ demande supérieure, comme le montre la figure 5.

Il est tout à fait surprenant que des tests purement psychologiques permettent de prévoir aussi bien les résultats à des examens traditionnels de chimie. Ceci implique que parmi les nombreux facteurs influant sur ta résolution de problèmes recensés par d'Hainaut (1983) la $\mathrm{M}$-capacité des élèves serait très largement dominante. Il est souhaitable que ces résultats soient confirmés par d'autres chercheur's dans des situations différentes. Les premiers résultats de J. Slootmaekers et col. (1989) montrent des courbes beaucoup plus douces et se rapprochent des résultats de Niaz.

Le fait que les performances ne tombent pas à zéro pour les étudiants (figure 5c) est expliqué par l'utilisation par certains étudiants de stratégies (qu'ils ont trouvées euxmêmes ou qu'ils ont apprises) qui leur permettent de dépasser leur niveau de performance attendu. D'autres explications pourraient être envisagées.

Quelles conclusions pratiques peut-on tirer de ces études, en ce qui concerne l'enseignement?

* Il importe d'évaluer soigneusement la M-demande des problèmes posés dans les examens et de s'interroger particulièrement sur la signification, pour l'apprentissage de la chimie, de questions de M-demande élevée.

* Pour l'apprentissage il est préférable de diminuer la Mdemande des problèmes, en gardant leur logique.

* Il est souhaitable de développer chez les élèves des stratégies qui leur permettent (un peu comme le font les experts) de diminuer la $\mathrm{M}$-demande des problèmes.

* Il est souhaitable de développer les liens entre les différents concepts qui devront être manipulés, comme l'a remarqué Kempa.

* II est souhaitable de jouer sur les motivations pour éviter qu'une partie seulement de la M-capacité soit employée.

\section{INFLUENCE DES MÉTHODES DEPATTERN RECOGNITION}

La chimie ayant à travailler sur des objets de plus en plus nombreux (environ 10 millions de composés chimiques sont actuellement connus) a dû développer des techniques de classification performantes. Les exemples historiques les plus célèbres sont la classification périodique et le système des fonctions en chimie organique. Des exemples plus récents et moins connus sont les règles de WoodwardHofmann et la théorie HSAB de Pearson.

Tout récemment une nouvelle discipline appelée chimiométrie par analogie avec la biométrie s'est donné pour objet de traiter les informations complexes dans tous les domaines de la chimie. Son domaine d'application est très vaste et comprend, par exemple, les relations structure-activité en pharmacologie aussi bien que l'optimisation des méthodes d'analyse.

Parailleurs l'avènement d'une informatique très performante et les énormes besoins industriels ont amené a créer de multiples bases de données qui ne sont encore que partiellement exploitées. Il était sans doute inévitable que ce courant important se traduise au niveau de l'enseignement et que soit enseigné le raisonnement par analogie qui irrigue une partie importante de la chimie.

Un des premiers enseigants chimistes à se lancer dans cette voie nouvelle a été Aleksandra Kornhauser, de Ljubljana en Yougoslavie. Je lui emprunte les deux exemples suivants qui illustrent sa démarche.

La structure générale de la chimie des sucres peut être redécouverte par les étudiants, à partir de la littérature, par analogie avec la chimie non hétérocyclique (figure 6).

Figure 6

Exemple de création d'une structure par les étudiants (d'après A. Kornhauser, 1984).

6a: Reconnaissance d'une structure simple

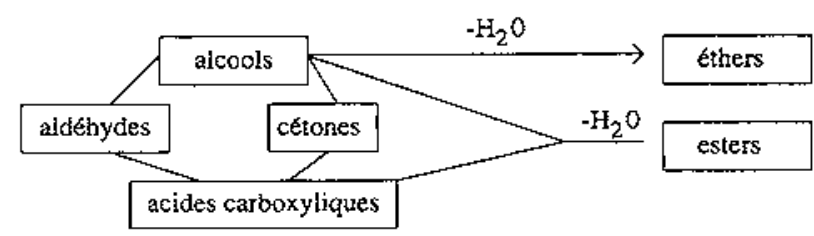

6b: Redécouverte d'une structure dons un autre domaine, par recherche d' analogies

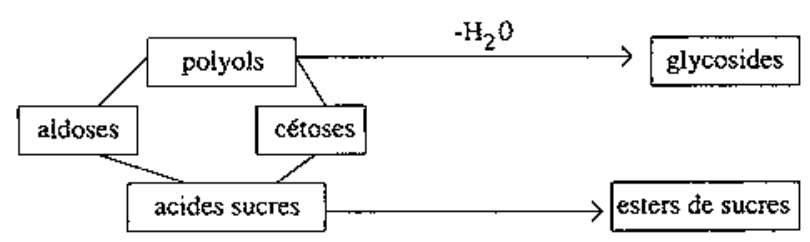

Un autre exemple montre le traitement d'une information hétérogène (brevets et publications) et permet de se rapprocher davantage de situations réelies de la chimie industrielle. Il s'agit de la synthèse d'un produit décrite 
dans plusieurs brevets et publications. Pour chacune des variables qui agissent sur la synthèse on peut effectuer un traitement semblable à celui qui est présenté pour la température (figure 7).

La comparaison des différentes gammes de température permet aux étudiants de proposer une température optimale de réaction (cas réel).

Figure 7

Recherche d'une température optimale de réaction (d'après A. Kornhauser, 1984).

Snirrer

Un autre exemple tout récent est encore plus explicite (Roy 1989). Il s'agit de faire découvrir aux étudiants (High School) des relations structure-activité pour les substances à goût sucré. Pour cela les molécules sont représentées à l'échelle convenable sur des transparents que les étudiants doivent superposer pour découvrir les régions favorables ou défavorables au goût sucré.

Un article récent de Kempa et Ward (1988) pose le problème très intéressant des seutls d'observation des phénomènes chimiques ou physiques dans le secondaire. Il s'agit encore, d'une certaine manière, de percevoir des informations pertinentes dans une situation complexe.

\section{LE RÔLE DU CTC DE L'IUPAC}

L'IUPAC (Union Internationale de Chimie Pure et Appliquée) est une association volontaire, non gouvernementale et à but non Iucratif. Fondée en 1919 , elfe regroupe 43 organisations dont chacune représente les chimistes d'un pays membre. Ces organisations nationales peuvent être un Comité National, un Conseil de la Recherche, une Académie des Scjences.

Environ 1500 chimistes à travers le monde sont engagés dans les travaux de I'IUPAC. Ils travaillent dans 30 commissions qui sont regroupées en 7 Divisions correspondant aux grands domaines de la Chimie (chimie minérale, organique, clinique, polymères). A côté de ces divisions on trouve plusieurs comités dont le Comité pour l'Industrie, le Comité pour l'Enseignement de Ia Chimie et le Comité Chemrawn (Chemical Research Applied to World Needs) qui ne sont pas rattachés à une Division particulière.
En dehors des membres des commissions et des Comités, l'IUPAC possède des membres associés qui peuvent être soit des sociétés industrielles (Company Associate Scheme) soit des individus (Affiliate Membership Scheme).

L'IUPAC joue un rôle déterminant pour fixer les conventions internationales (nomenclature, unités) et publie régulièrement des règles bien suivies par la communauté des chimistes. Des recueils de données des comptes rendus de congrès sont également publiés.

L'IUPAC est membre de l'ISCU (International Council of Scientific Unions) et collabore, en ce qui concerne la chimie, avec les grandes organisations internationales telles que WHO (World Health Organization), UNEP, FAO et ISO (International Organization for Standarization).

Le CTC (Committee on Teaching of Chemistry) a pour mission de traiter tous les aspects de l'enseignement de la chimie. Il est représenté dans le CTS (Committee on Teaching of Science) de I'ICSU. En dehors de ses associations-mères le CTC entretient une collaboration étroite avec l'UNESCO dont il reçoit une aide importante dans le cadre d'actions internationales ou nationates.

Dans les dix dernières années le CTC a eu une action soutenue en direction de l'enseignement secondaire et plus spécifiquement de la formation des maîtres.

L'action la pius importante par son ampleuret sa philosophie est certainement le projet UNESCO/IUPAC-CTC concernant le matériel d'enseignement produit localement et à bon marché.

Ce projet a été lancé vers 1979 par K. Sané, actuellement Secrétaire du CTC, et D. Waddington (ancien Président) avec le soutien sans faille de l'UNESCO. Il cherchait à répondre aux grandes difficultés rencontrées pour l'enseignement expérimental de la chimie dans les pays en développement où l'on trouve des classes très nombreuses, avec un équipement rare et une maintenance insuffisante. Les objectifs poursuivis étaient les suivants:

- développer deséquipements fiables, bon marché, produits avec les composants locaux, faciles à fabriquer et à entretenir;

- préparer un ensemble d'expériences compatibles avec ces matériels et illustrant la chimie moderne;

- transférer les compétences à un nombre élevé d'enseignants, au moyen d'ateliers de prodution, de livres et autres documents;

- mettre sur pied un système de production à une échelle importante (plusieurscentaines d'unités) sans augmentation importante des coûts.

Le groupe de l'Université đe Delhi a organisé plus de 30 ateliers et formé environ 600 professeurs. Un millier d'appareils ( $\mathrm{pH}$ mètres, colorimètres, conductimètres et leurs accessoires, agitateurs magnétiques, etc.) ont été fabriqués pour des prix allant de 20 à 50 USD et sont utiłisés dans de nombreuses Universités et établissements secondaires. 
Des groupes indépendants.ont été formés à partir de ce noyau initial et déploient une grande activité en Australie, au Bangladesh, en Thailande, au Brésil, à Porto Rico, en Jordanie et dans d'autres pays. Je tiens à souligner que le réseau mis en place vise à rendre les professeurs aussi autonomes que possible et relève d'une approche complètement opposée à certains aspects de «l'aide au Tiers Monde».

Depuis quelques années le CTC aide au développement d'un réseau francophone basé sur des idées voisines mais couvrant une plus grande variété d'actions. Ce réseau est animé par le CIFEC (Centre International Francophone pour l'Education en Chimie), dirigé par D. Cros, de l'Université de Montpellier. On retrouve les idées fondamentales d'autonomie des enseignants, d'utilisation des ressources locales et de formation associée à la production.

A côté des matériels décrits ci-đessus le CIFEC produit des ensemble à très faible coût pour la réalisation d'expériences sur de très petites quantités, ce qui limite les coûts et les problèmes de sécurité. Ces ensembles sont déjà utilisés dans les lycées dans le sud de la France et en Tunisie par exemple.

Parallèlement le CIFEC a un vaste programme de formation de formateurs, pour ia conception et l'evaluation des programmes et des manuels dans les pays francophones.

Dans toutes ces actions on recherche un effet de boule de neige qui est obtenu assez souvent en dépit des conditions difficiles rencontrées dans certains pays.

Un autre rôle du CTC est de favoriser la cohésion de la communauté des enseignants de chimie. Il organise tous les deux ans un Congrès international (International Conference on Chemical Education) qui réunit 500 à 700 enseignants d'une cinquantaine de pays. Ces congrès ont un rôle d'entrainement dans les pays qui les organisent. Entre les Congrès, des Symposium sont organisés à l'occasion des réunions annuelles du CTC (à Rome en 1986, Lisbonne en 1988 et Moscou en 1990).

Ces Symposium permettent à 200 à 300 professeurs de recontrer entre autres les membres du CTC et les représentants nationaux au CTC.

Un lien d'une autre sorte est assuré par la publication d'un journal (International Newsletter on Chemical Education) distribué gratuitement à 3000 exemplaires dans le monde entier. Ce journal qui paraît deux fois par an présente surtout des innovations et des réalisations et seulement exceptionnellement des résultats de recherches.

Le CTC a plusieurs autres types d'activité tels que l'organisation d'ateliers, une contribution aux Olympiades Internationales de Chimie, la publication de livres avec l'Unesco, etc.

Tout récemment l'IUPAC a demandé au CTC de superviser toutes les activités de l'association en relation avec l'enseignement. Il est demandé au CTC d'augmenter ses activités au niveau universitaire tout en poursuivant ses actions au niveau secondaire et en direction du grand public. Ceci implique une réorganisation qui est en cours. L'un des objectifs fixés est d'assurer le passage rapide et correct au niveau de l'enseignement des progrès réalisés sur les frontières de la chimie.

Ceci n'est d'ailleurs pas propre à la seule chimie et le problème est abordé aussi au niveau de l'ICSU-CTS. D'après les règles établies pour utiliser les résultats du programme Global Change de l'ICSU.

Les documents produits doivent:

- être compatibles avec les programmes

- être courts

- illustrer des principes scientifiques importants

- ne pas demander trop de temps d'enseignement

- être d'un emploi aisé

- être bon marché à la production et à l'emploi

- être interactifs

- être adaptables aux conditions locales

Les documents peuvent être produits sous la forme de petits livres pour les enseignants, contenant:

- une information scientifique de qualité, préparée par le CTS en collaboration avec les scientifiques,

- des stratégies d'enseignement détaillées

- un système d'évaluation.

Il a paru très important que ces livres soient non seulement traduits mais aussi adaptés en fonction des besoins d'un pays particulier.

Par contre le lien entre l'enseignement tel qu'il est généralement assuré et la recherche en Didactique de la Chimie est loin d'être satisfaisant. Le Comité a commencé à travailler dans le sens d'une meilleure utilisation de la recherche en visant à instaurer une sorte de veille scientifique et technologique.

\section{CONCLUSION}

J'espère avoir montré sur des exemples précis quelques problèmes rencontrés par la Didactique de la Chimie. Les tendances que j'ai soulignées ne sont pas les seules et cette analyse ne représente que mon opinion personnelle.

Le Comité pour l'Enseignement de la Chimie de l'IUPAC soutient ou provoque des actions visant à améliorer la formation des professeurs, les pratiques et les matériels d'enseignement et la prise en compte des relations entre la Science, la Technologie et la Société. L'ampleur de la tâche à accomplir en particulier dans les pays en développement rend précieux tous les progrès faits dans les dix dernières années. 


\section{RÉFÉRENCES BIBLIOGRAPHIQUES}

D'HAINAUT, L., 1983. Des fins aux objectifs, 3ème édition. (Labor/Nathan, Bruxelles/Paris).

FRAZER, M. J. et SLEET, R.J., 1984. A study of students' attempts to solve chemical problems, European Journal of Science Education, 6 (2), pp. 141-152.

FURIO, C. et HERNÁNDEZ,J., 1987. La resolución superficial de problemas de Química: un ejemplo estequiométrico, Enseñanza de las Ciencias, extra, pp. 199-200.

GIL, D. et MARTÍNEZ TORREGROSA, J., 1983. A model for problem solving in accordance with scientific methodology, European Journal of Science Education, 5, pp. 447-455.

IOHNSTONE, A. H. et EL BANNA, H., 1986. Capacities, demands and processes - a predictive model for science education, Education in Chemistry, pp. 80-83.

KEMPA, R. F. et WARD, J. E., 1988. Observational thresholds in school chemistry, International Journal of Science Education, 10 , pp. 275-284.

KORNHAUSER, A., 1984. Understanding Science, en Teaching and Popularizing Science and Technology, Kornhauser A. and Richardson J. G. (Unesco, Ljubljana), pp. 97-116.

LAZONBY, J,N., MORRIS, J.E. et WADDINGTON, D.J., 1982. Calculations involving the mole concept. An enquiry into difficulties experienced by pupils. Proceedings of the 6th. International Conference on Chemical Education. (Maryland, 1981). W.T. Lippincott Ed., 178.
NIAZ,M, 1988. The information-processing demand of chemistry problems and its relation to Pascual-Leone's functional Mcapacity, International Journal of Science Education, 10 , pp. 231-238.

NÍAZ, M., 1989. Relation between Pascual-Leone's structural and functional $M$-space and its effect on problem solving in chemistry, International Journal of Science Education, 11 , pp. 93-99.

PEREIRA, M. P. B. A., 1986. Equilibrio Químico. Dificuldades de Aprendizagem e Sugestoes Didacticas. (Universidade de Lisboa).

PEST ANA, M. E., 1989. Teachers and Students' Strategies in chemical problem solving. Abstracts of the 10th. (I.C.C.E. Waterloo. Canada). August 20 th. to 25 th. 1989.

POLICH, J.M. et SCHWARTZ, S.H., 1974. The effect of problem size on representation in deductive problem solving, Memory and Cognition, 2, pp. 683-686.

ROY, G., 1989. A Simple qualitative technique for Pattern Recognition in Structure-Activity Relationships, Journal of Chemical Education, 66 (5), pp. 435-436.

SLOOTMAEKERS,P.J.et jOHNSTONE, A.H., 1989. Academic Performance in solving chemistry problems related to student working memory capacity, Abstracts of the 10th. I.C.C.E. Waterloo. Canada. August 20 th. to 25 th. 1989.

\section{RESUMEN}

Según el autor, la didáctica de la Química ha experimentado un gran desarrollo en los últimos tiempos y se encuentra en una fase de expansión rápida, por lo que su trabajo no representa más que una visión particular y parcial de esta temática. La visión que se presenta engloba el interés del autor y el desarrollo ofrecido por el Comité para la Enseñanza de la Química (CTC) de la Unión Internacional de Química Pura y Aplicada (IUPAC).

Para el autor pueden distinguirse dos tendencias: la primera es la intemacionalización de las investigaciones en didáctica y de las innovaciones significativas y la segunda es la diversificación de los campos estudiados y de los métodos utilizados.

Hace dos décadas las reflexiones y las innovaciones se centraban en "los contenidos" de la enseñanza, mientras que ahora a la controversia sobre los contenidos se ha añadido el interés por el propio proceso de aprendizaje. Este interés se ha traducido en un buen número de publicaciones sobre las "representaciones" de los alumnos, sobre la resolución de problemas, sobre el tratamiento de la información, sobre la significación de las prácticas... El concepto de equilibrio en química ha sido tomado por el autor como ejemplo de las investigaciones acerca de las representaciones.

En el tema de la resolución de problemas el autor señala que la aproximación de los químicos es, con frecuencia, muy utilitaria y muestra una gran diversidad. Se pasa revista a las aportaciones de varios autores y se insiste en el aspecto relativo a las capacidades para la resolución de problemas, a la luz de las publicaciones sobre la relación entre dificultad del problema, capacidades requeridas y medida de estas capacidades.

Se han considerado también de forma somera publicaciones sobre la influencia de métodos de reconocimiento de modelos (pattern recognition).

Finalmente se señalan algunos de los proyectos más importantes desarrollados por el Comité para la Enseñanza de la Química, sobre todo los orientados a resoiver las dificultades para la ensenanza experimental de la química en los países en desarrollo, con clases numerosas, con equipo escaso y mantenimiento insuficiente. 